International Journal of Bifurcation and Chaos, Vol. 13, No. 3 (2003) 609-616

(c) World Scientific Publishing Company

\title{
CLOSED CURVES OF GLOBAL BIFURCATIONS IN CHUA'S EQUATION: A MECHANISM FOR THEIR FORMATION
}

\author{
ANTONIO ALGABA and MANUEL MERINO \\ Department of Mathematics, E. Politécnica Sup., Univ. Huelva, \\ Crta. Palos-Huelva s/n, 21819 La Rábida, Huelva, Spain \\ FERNANDO FERNÁNDEZ-SÁNCHEZ and ALEJANDRO J. RODRÍGUEZ-LUIS \\ Department of Applied Mathematics II, E.S. Ingenieros, Univ. Sevilla, \\ Camino de los Descubrimientos s/n, 41092 Sevilla, Spain
}

Received February 14, 2002; Revised March 22, 2002

\begin{abstract}
In this work, the presence of closed bifurcation curves of homoclinic and heteroclinic connections has been detected in Chua's equation. We have numerically found and qualitatively described the mechanism of the formation/destruction of such closed curves. We relate this phenomenon to a failure of transversality in a curve of T-points in a three-dimensional parameter space.
\end{abstract}

Keywords: Global bifurcations; T-point; Chua's equation.

\section{Introduction}

An important task for the understanding of the dynamics of parameterized systems of autonomous ordinary differential equations is the determination of the organizing centres as well as the bifurcations they exhibit (see, e.g. [Guckenheimer \& Holmes, 1983; Kuznetsov, 1995; Nayfeh \& Balachandran, 1995; Wiggins, 1996] as general references). The combination of analytical and numerical tools is usually needed due to the existence of a complex bifurcation scenario. To complement theoretical results, numerical continuation is done in one parameter (bifurcation diagram, for example, period versus parameter) or in two (or more) parameters (bifurcation set, loci where bifurcations occur in the parameter space).

The presence of isolas (isolated closed curves of solution branches) in bifurcation diagrams of periodic orbits has been detected, for example, in relation to Hopf curves (see, e.g. [Doedel et al., 1991]). The appearance of an isola configuration depends on the choice of the bifurcation parameter as well as on the shape of the bifurcation curve.

A typical o-shaped isola of periodic orbits appears when moving inside resonance zones close to the tip where the corresponding Arnold's tongues emerge (see, e.g. Fig. 12(a) in [Algaba et al., 2001]).

Other kind of isolas of a certain type of periodic orbits was detected, in an electronic circuit, by [Fernández-Sánchez et al., 1997]. In that work, the mechanism of their formation is shown and their existence is related to cusp bifurcations and to Shil'nikov homoclinic connections.

In other works, some kinds of isolas have appeared in relation with homoclinic bifurcations/tangencies. In this way [Hirschberg \& Laing, 1995] have shown that primary periodic orbits lie on an infinity of isolas in a neighborhood of a homoclinic tangency to a periodic orbit. Also [Champneys \& Rodríguez-Luis, 1999] have found, unfolding a nontransverse Shil'nikov-Hopf bifurcation, 
finitely and infinitely many isolas of periodic orbits. They try to explain in a concrete process by which loci of homoclinic orbits and homoclinic tangencies can become disconnected and to show that this naturally leads to isolas of periodic orbits in the parameter regimes of both homoclinic orbits and homoclinic tangencies.

On the other hand, the continuation of a bifurcation curve in a parameter plane usually leads to the detection of higher codimension bifurcations (sometimes the endpoints of the curve, other times special points on the curve where a nondegeneracy condition fails). However, the existence of a closed bifurcation curve is less common. In this rare situation, normally some nontransversality with respect to other parameter occurs (due to the shape of the corresponding bifurcation surface in the three-parameter space) and then the presence of a codimension-two bifurcation can be suspected. For instance, closed curves of saddle-node bifurcations of periodic orbits have been detected in [Fernández-Sánchez et al., 1997] in a scenario with cusps and homoclinic connections. Closed curves of torus bifurcation were found in [Algaba et al., 2001] in connection with two Takens-Bogdanov bifurcations of periodic orbits.

In this work we focus on global connections (homoclinic and heteroclinic orbits to equilibria). In fact, we numerically find, in a parameter plane, closed curves of global connections in Chua's equation, one of the dynamical systems most widely studied in the last two decades, a prototype of $\mathbb{Z}_{2}$-symmetric systems (invariant under the change of the sign in the state variables, $(x, y, z) \rightarrow$ $(-x,-y,-z))$. Recall that homoclinic/heteroclinic orbits to equilibria can originate complicated recurrent dynamics (see, e.g. [Guckenheimer \& Holmes, 1983; Kuznetsov, 1995] for summaries).

Moreover, we describe the mechanism of the formation of such closed curves and we relate them to the presence of a curve of T-points in a three-dimensional parameter space. A T-point is a codimension-two heteroclinic connection where the one-dimensional manifolds of two saddle equilibria are connected and the loop is closed with an intersection of the two-dimensional manifolds (see [Glendinning \& Sparrow, 1986; Bykov, 1993; Fernández-Sánchez et al., 2002]).

The paper is organized as follows. In Sec. 2, we introduce the system considered, namely Chua's equation. We explain the numerical process followed to find closed bifurcation curves of global con- nections and we describe the mechanism for the creation/destruction of these closed curves. Section 3 contains some conclusions and suggestions for future work.

\section{Numerical Study}

Let us start by introducing Chua's equation, that models a simple electronic circuit exhibiting a wide range of complex dynamical behaviors. Such equation is derived in [Pivka et al., 1996] considering the case of a cubic nonlinearity:

$$
\begin{aligned}
& \dot{x}=\alpha\left(y-a x^{3}-c x\right), \\
& \dot{y}=x-y+z, \\
& \dot{z}=-\beta y-\gamma z .
\end{aligned}
$$

In this equation a parameter $\gamma$ is included in order to take into account small resistive effects in the inductance of the circuit.

In our numerical study of Chua's equation (1), performed basically with AUTO97 [Doedel et al., 1998], we will take $\beta, c$ and $\alpha$ as bifurcation parameters and we will fix the other two, namely $a=-1$, $\gamma=0.3>0$, in accordance to other previous works (see [Algaba et al., 2000, 2003]).

First, we focus on a codimension-two local bifurcation (Takens-Bogdanov) that it is well known that leads to the presence of global bifurcations. In Fig. 1, for $\alpha=-1.342$, we represent the partial bifurcation set in the vicinity of the TakensBogdanov bifurcation of the origin $\mathbf{T B}$, that occurs for $\beta \approx 0.01572, c \approx 0.9502$. Note that the values where a nondegenerate Takens-Bogdanov bifurcation occurs in Eq. (1) can be analytically deduced from the equations (see [Algaba et al., 2003]):

$$
\alpha(\gamma-c \beta-c \gamma)=0, \quad \alpha-c \alpha-c \alpha \gamma-\beta-\gamma=0
$$

with the nondegeneracy condition

$$
(c \alpha+\gamma+1) \neq \frac{\beta+\gamma}{\gamma+1} .
$$

Moreover, the Takens-Bogdanov bifurcation is of homoclinic type when

$$
(c \alpha+\gamma+1) \sqrt[3]{\frac{a \alpha}{\beta^{2}}}(\beta+\gamma)>0,
$$

condition that is satisfied by the parameter values considered above. The point TB occurs at the intersection between a pitchfork curve PI and a Hopf bifurcation curve Ho of the origin (this one is, for 


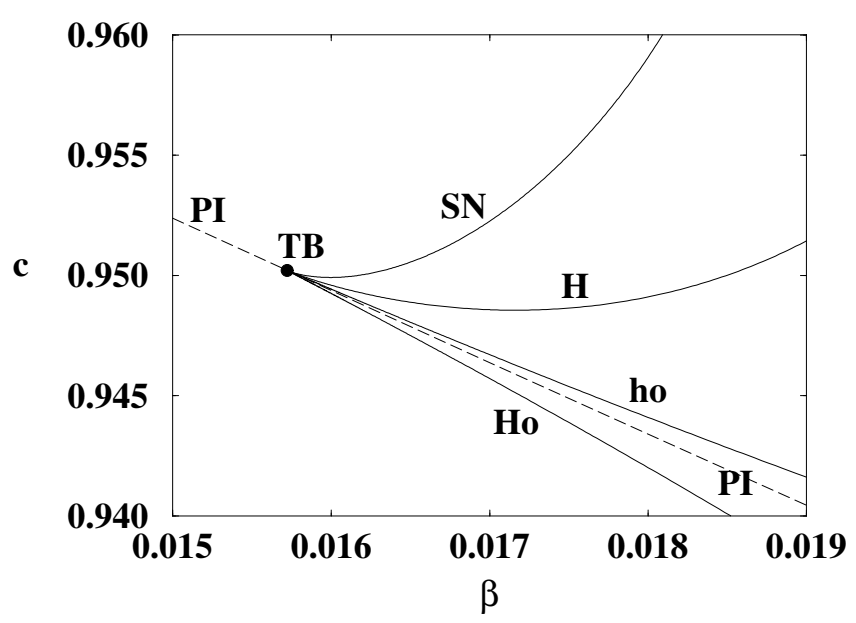

Fig. 1. Partial bifurcation set, for $\alpha=-1.342, \gamma=0.3$ and $a=-1$, in the region where the Takens-Bogdanov bifurcation of the origin TB appears. The curves corresponding to a pitchfork bifurcation of the origin PI, a subcritical Hopf bifurcation of the origin Ho, a saddle-node bifurcation of periodic orbits $\mathbf{S N}$, a homoclinic orbit to the origin $\mathbf{H}$ and a supercritical Hopf bifurcation of the nontrivial equilibria ho are drawn.

these values of the parameters, subcritical; this means that the symmetric periodic orbit emerged is nonstable).

Moreover, three new curves emerge from a Takens-Bogdanov point of homoclinic type (see, e.g. [Guckenheimer \& Holmes, 1983; Kuznetsov, 1995]): ho, a curve where the nontrivial equilibria undergo a supercritical Hopf bifurcation (and then a pair of stable asymmetric periodic orbits emerges); $\mathbf{H}$, a curve of homoclinic connections of the origin; and $\mathbf{S N}$, corresponding to saddle-node bifurcations of symmetric periodic orbits.

The curve of principal homoclinic connections $\mathbf{H}$, that emerges from the point $\mathbf{T B}$ when the origin is a saddle equilibrium, presents some degeneracies not deduced from the analytical study of local bifurcations inside the parameter range shown in Fig. 1. The first one is caused by the transition of the origin from saddle to saddle-focus, for $\beta \approx 0.01573, c \approx 0.95017$. Since the double eigenvalues are not determining (an eigenvalue is called determining if it is the eigenvalue closest to the imaginary axis) the homoclinic bifurcation remains tame (see, e.g. [Champneys \& Kuznetsov, 1994]). If we denote by $(\rho \pm i \omega, \lambda)$ the eigenvalues of the trivial equilibrium, the second degeneration on $\mathbf{H}$ that occurs for $\beta \approx 0.01592, c \approx 0.94975$ is produced as $\delta=|\rho / \lambda|=1$ (resonant eigenvalues). The transition from tame to chaotic homoclinic orbit occurs at this point, where it enters into the socalled Shil'nikov region.

According to the results of [Glendinning, 1984], because of the $\mathbb{Z}_{2}$-symmetry Chua's equation exhibits, the symmetric periodic orbit that emerges from the curve Ho will undergo several bifurcations as it approaches the principal homoclinic connection $\mathbf{H}$, in the region where the Shil'nikov condition $\delta<1$ is satisfied [see Fig. 2(a)]. In fact, the symmetric periodic orbit experiments pitchfork PPO and saddle-node SN bifurcations. The first saddle-node $\mathbf{S N}$ (that is on the $\mathbf{S N}$ curve emerged from TB in Fig. 1) and the first pitchfork PPO are shown in Fig. 2(a).

To detect secondary homoclinic connections we continue the asymmetric periodic orbits emerged from the point PPO [dashed branch in Fig. 2(a)]. A pair of asymmetric periodic orbits, Axz and its symmetric Azx, is born from the point PPO. They are characterized because their projections onto the $x-z$ plane surrounds one time to each nontrivial equilibrium, $P^{+}(x, y,-z)$ and $P^{-}(-x,-y, z)$, where we consider $x>0, y>0$ and $z>0$. Both orbits disappear in another pair of double-pulse homoclinic connections, $\mathbf{H x z}$ (the projection onto the $x-z$ plane of the one-dimensional manifold of the equilibrium surrounds first $P^{+}$and then $P^{-}$) and its symmetric $\mathbf{H z x}$ (the projection onto the $x-z$ plane of the one-dimensional manifold of the equilibrium surrounds once first $P^{-}$and then $P^{+}$). From now on, for the sake of simplicity, we will only mention one homoclinic of each pair (for instance, in the case of the symmetric pair $\mathbf{H x z}$ and $\mathbf{H z x}$ we will only write $\mathbf{H x z}$ ).

The numerical continuation of the symmetric periodic orbit [see Fig. 2(a)], that also wiggles around the homoclinic connection $\mathbf{H x z}$ (see [Glendinning, 1984]), shows that such periodic orbits do not emerge from a local bifurcation but they connect two global connections: The homoclinic connection $\mathbf{H x z}$ and the heteroclinic connection $\mathbf{H e}$ both located inside the Shil'nikov region. The projections onto the $x-z$ plane of the pair of heteroclinic connections and of the homoclinic orbits to the origin $\mathbf{H}$ and $\mathbf{H x z}$ are shown in Figs. 2(b)-2(d), respectively.

The continuation of the curve $\mathbf{H e}$ in the $(\beta, c)$ parameter plane (see Fig. 3) shows that it is a closed curve. Apparently, for these values of the parameters, $\mathbf{H e}$ is not connected with a codimension-two local or global bifurcation. Thus, we are going to move the third parameter, $\alpha$, to see the evolution of 


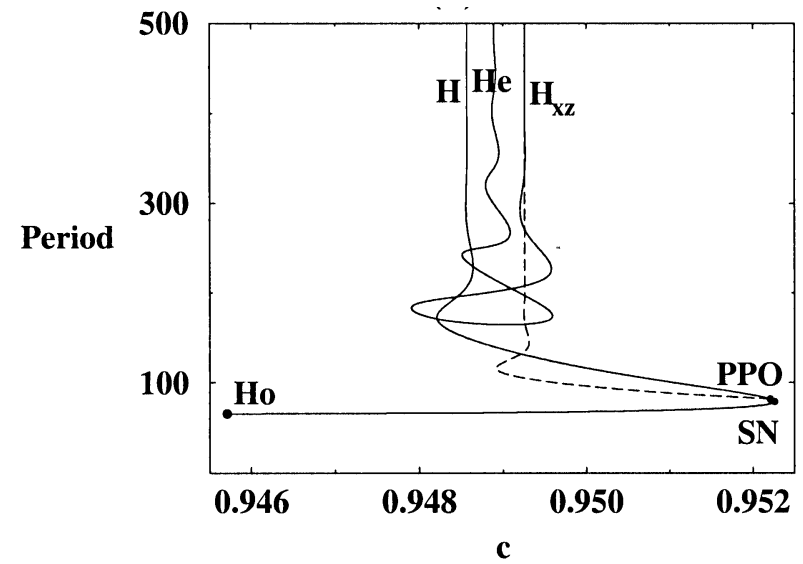

(a)

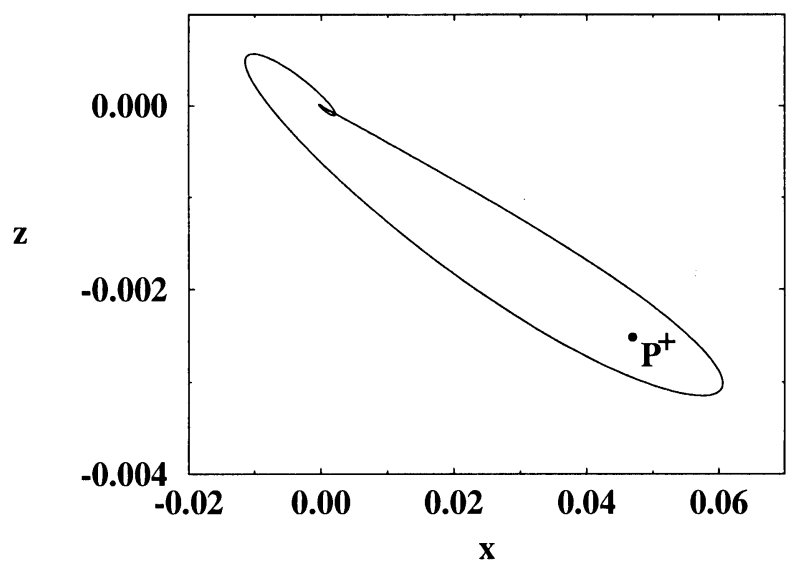

(c)

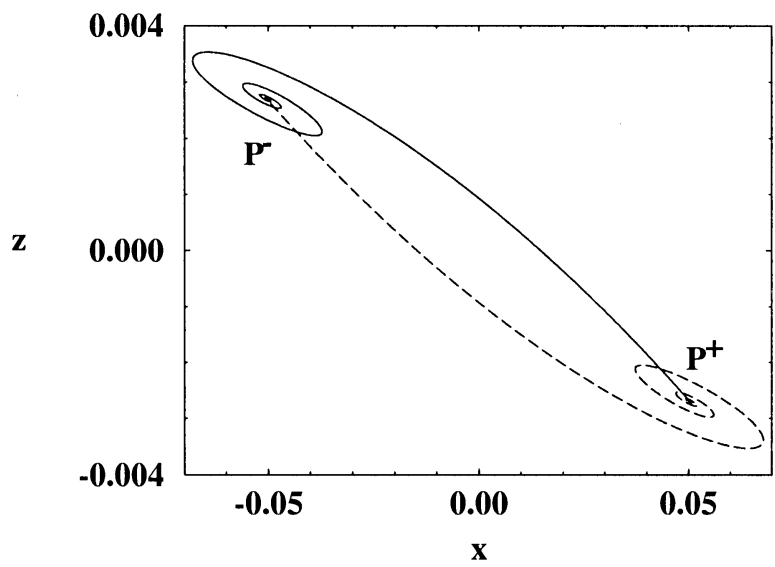

(b)

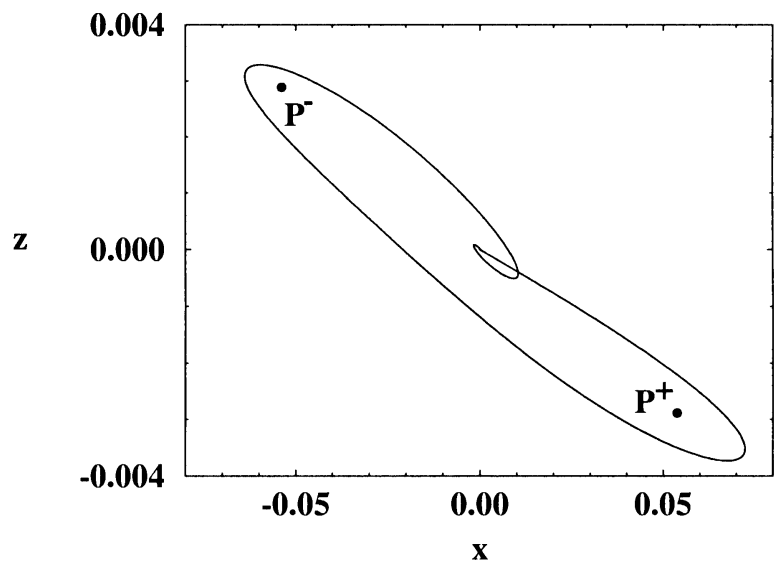

(d)

Fig. 2. For $\beta=0.017, \alpha=-1.342, \gamma=0.3, a=-1$ : (a) Partial bifurcation diagram ( $c$ versus period) of the symmetric (solid line) and asymmetric (dashed line) periodic orbits. (b) Projection onto the $x-z$ plane of the pair of heteroclinic connections He (one appears as a solid line and the other as a dashed one) that occurs for $c \approx 0.9489$. (c) Projection onto the $x-z$ plane of the homoclinic connection $\mathbf{H}$ that occurs for $c \approx 0.9486$. (d) Projection onto the $x-z$ plane of the homoclinic connection $\mathbf{H x z}$ that occurs for $c \approx 0.9493$.

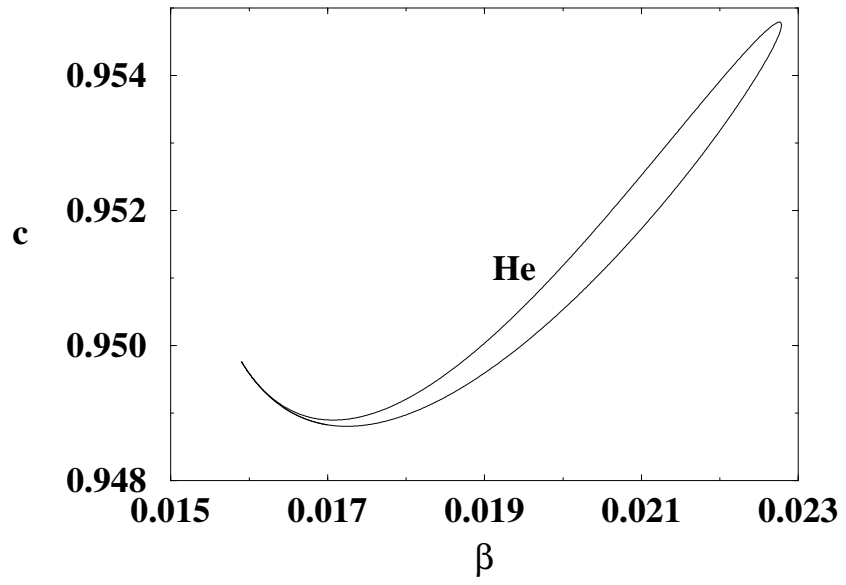

Fig. 3. Closed bifurcation curve of heteroclinic connections He for $\alpha=-1.342, \gamma=0.3, a=-1$. the He curve and to investigate the presence of new bifurcation points that act as organizing centres of the heteroclinic connection He.

In this way, for $\alpha=-1.35$, it is shown in Figs. 4(a) and 4(b), that the heteroclinic connections curve $\mathbf{H e}$ is not already closed and that its end-points correspond to two different T-points TP. Note that, in a three-dimensional system, having at least two saddle equilibria, a T-point occurs when the one-dimensional unstable manifold of one of the equilibria and the one-dimensional stable manifold of the other equilibrium coincide. At the same time the two-dimensional manifolds of the equilibria have a transversal intersection originating a heteroclinic loop (set of two heteroclinic orbits) 


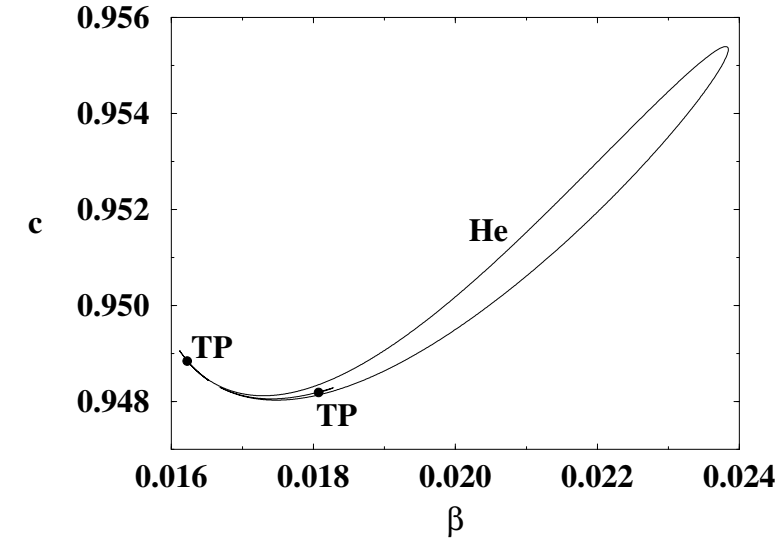

(a)

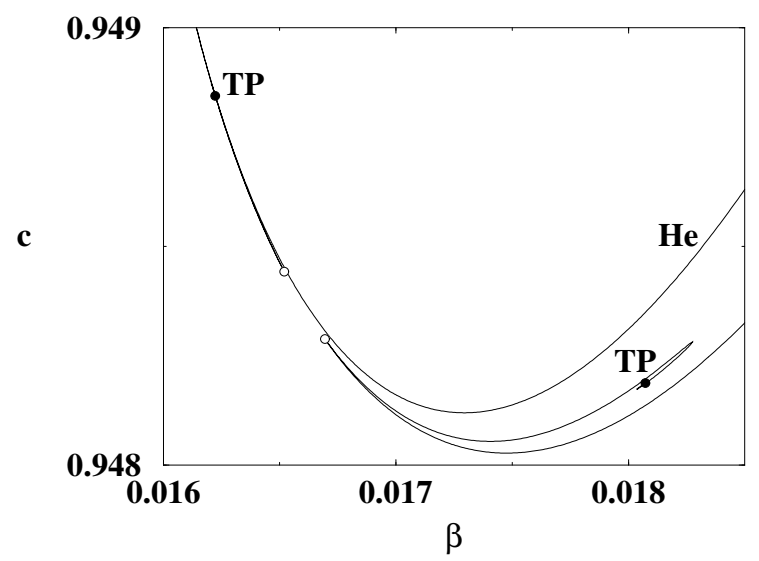

(b)

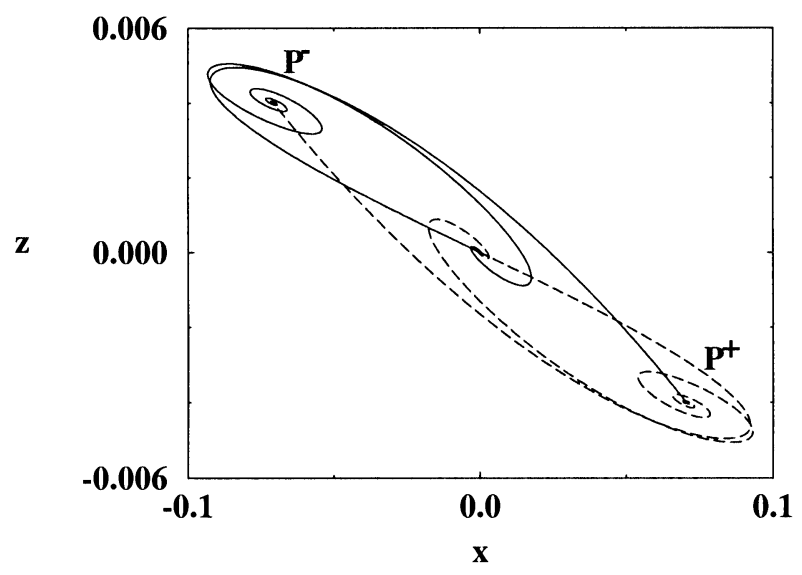

(c)

Fig. 4. For $\alpha=-1.35, \gamma=0.3, a=-1$ : (a) Heteroclinic connection curve He joining two T-points TP. (b) Details of the above curve in the region where the T-points $\mathbf{T P}$ occur to show that it is not closed. (c) Projection onto the $x-z$ plane of the pair of T-points (the solid line corresponds to a heteroclinic entering to the T-point and the dashed line to its symmetric one) that occurs for $\beta \approx 0.01807, c \approx 0.94819$ [right TP point in (a) and (b)].

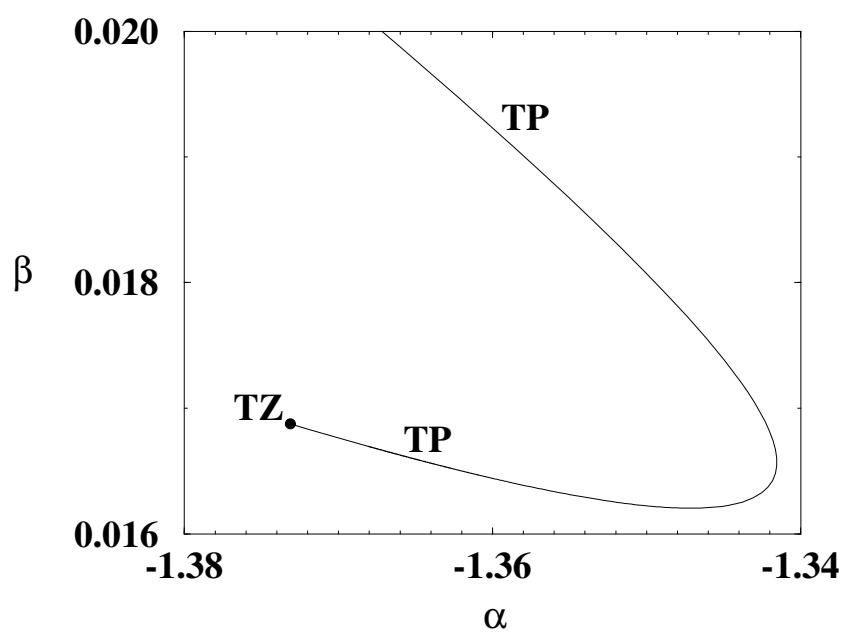

Fig. 5. Projection of the curve of T-points TP onto the $(\alpha$, $\beta$ ) plane, for $\gamma=0.3, a=-1$. This curve emerges from a triple-zero linear degeneracy of the origin TZ. A fold appears for $\alpha_{c} \approx-1.34156$.

between both equilibria. Usually, this codimensiontwo heteroclinic loop is also called T-point. We refer for details to [Fernández-Sánchez et al., 2002] and the references inside.

The projection of the heteroclinic pair onto the $x-z$ plane allows to see the shape of the pair of T-points [see Fig. 4(c)] that occurs for $\beta \approx 0.01807$ [right TP point in Fig. 4(b)].

To analyze these new codimension-two global bifurcations, we continue, in the $(\alpha, \beta, c)$-parameter space, the curve of these T-points, TP. The projection of such curve onto the $(\alpha, \beta)$ plane is shown in Fig. 5. We can see that this curve emerges from a triple-zero linear degeneracy point of the origin TZ, that exists for $\alpha=-1.373125, \beta=0.016875$ and $c \approx 0.946746$ (see [Algaba et al., 2003] for details). Since the TP curve has a fold, it exhibits a transversality failure with respect to $\alpha$ (a maximum occurs for $\left.\alpha_{c} \approx-1.34156\right)$.

Consequently, for the first value of $\alpha$ considered, $\alpha=-1.342$, not only the closed curve of heteroclinic connections He shown in Fig. 3 is present but also a new branch of $\mathbf{H e}$ connecting the two Tpoints placed on the curve TP. This new curve is inside the closed one that is represented by a dashed line [see Fig. 6(a)].

If we compare Figs. 4(b) and 6(a) we observe how the turning points marked with empty bullets in Fig. 4(b) approach as $\alpha$ is increased towards $\alpha_{c}$ (value for which the turning point in the curve TP occurs). When the empty bullet points collapse a closed curve of heteroclinic connections is formed 


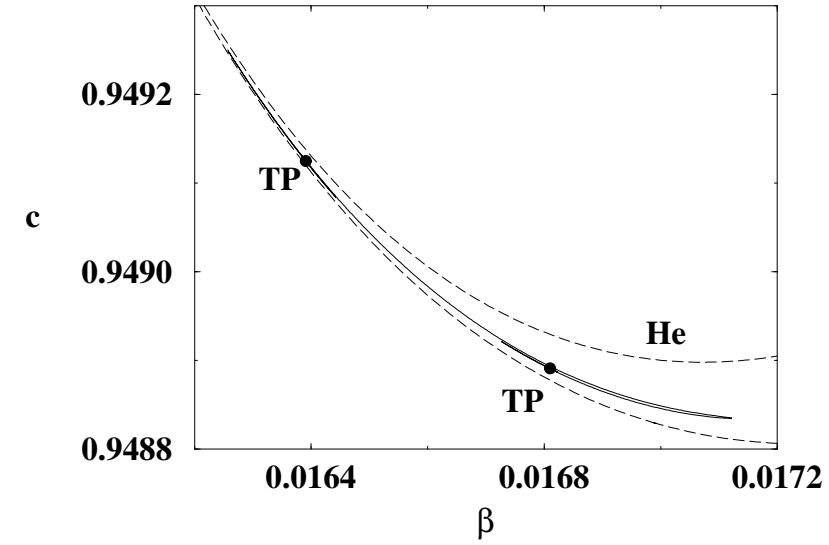

(a)

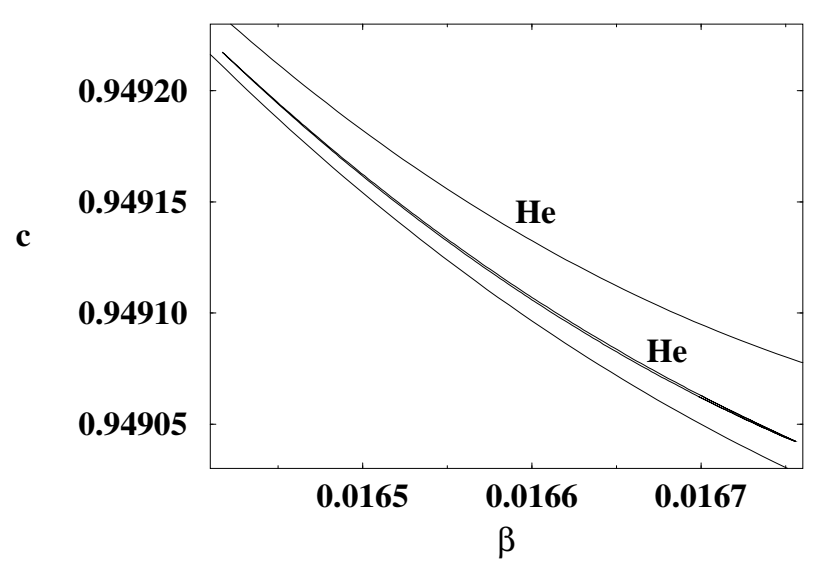

(b)

Fig. 6. For $\gamma=0.3, a=-1$ : (a) Two curves of heteroclinic connections He for $\alpha=-1.342$. The inner one (solid) connects both T-points TP; the outer one (dashed) is closed (see Fig. 3). (b) Two closed curves of heteroclinic connections He for $\alpha=-1.3405$ (only a part of the outer one appears in the window shown).

(dashed line in Fig. 6(a), solid line in Fig. 3) and a new branch of heteroclinic connections organized by two T-points of the curve $\mathbf{T P}$ remains inside it.

For the sake of clarity, we show a qualitative picture in Fig. 7 that explains how the closed curves appear in the parameter plane $\beta-c$ when the parameter $\alpha$ moves close to $\alpha_{c}$. Note that this mechanism would be difficult to follow with the real curves $\mathbf{H e}$ in the numerical bifurcation set.

Situation 1 appears when the curve He connects both T-points (it emerges from them spiralling, since the origin and the nontrivial equilibria are saddle-focus equilibria; see [Fernández-Sánchez et al., 2002] for a theoretical explanation of this fact). In Chua's equation it corresponds, for instance, to $\alpha=-1.355$.

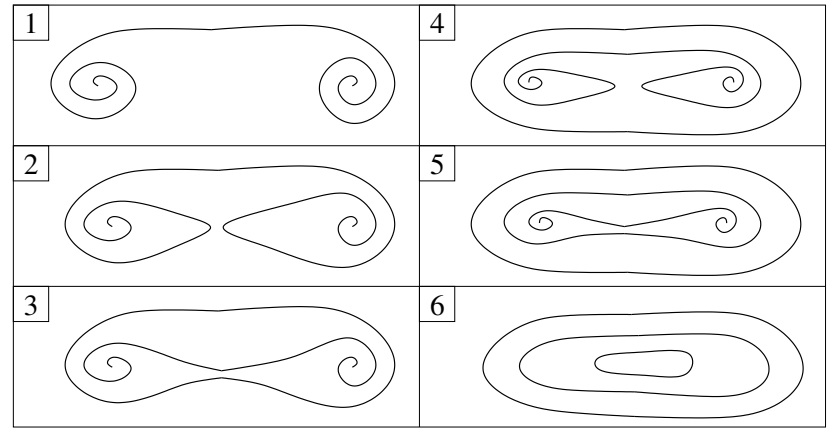

Fig. 7. Qualitative scheme of the evolution in the $(\beta, c)$ plane of heteroclinic curves in Chua's equation, when $\alpha$ is increased in a neighborhood of $\alpha_{c}$, value for which the T-point curve has a fold. Initially (1), there is only one heteroclinic curve spiralling around both T-points. In (2) the outer parts of both spirals approach and finally collapse (3) giving rise to a closed curve of heteroclinic orbits which contours the other branch connecting both T-points. As $\alpha$ is further increased, the same process is repeated for the formation of a second closed curve (situations 4-5). The formation of more closed curves continues till $\alpha_{c}$, where both T-points collapse in the $(\beta, c)$ plane. Finally, the closed bifurcation curves successively disappear. The last three closed curves are sketched in (6).

As $\alpha$ is increased (situation 2), the outer parts of both spirals approach. This occurs in Chua's equation, for example, when $\alpha=-1.35$. Immediately (situation 3), for $\alpha=-1.349$ in Chua's equation, after the contact between both spirals, a closed curve of heteroclinic orbits appears and, inside it, the other branch connecting both T-points coexists.

As $\alpha$ is further increased, the same process is repeated for the formation of a second closed curve (situations 4 and 5). The formation of more closed curves continues till $\alpha_{c}$, where both T-points collapse in the $\beta-c$ plane. When the turning point of the TP curve is crossed (for $\alpha_{c} \approx-1.34156$ ) there will not appear any T-point in the bifurcation set but the closed bifurcation curves still exist (we conjecture that an infinity of them exist for $\left.\alpha_{c}\right)$. Finally, increasing $\alpha$, the closed bifurcation curves successively disappear (firstly the inner ones). This is illustrated in situation 6 where only the three last closed curves remain. For instance, in Fig. 6(b), the two last closed curves corresponding to $\alpha=-1.3405$ in Chua's equation are drawn.

It is known (see [Fernández-Sánchez et al., 2002]) that from a T-point in a $\mathbb{Z}_{2}$-symmetric system two kinds of homoclinic connections also emerge: One connecting the origin and the other connecting each nontrivial equilibrium. We have checked that, with an analogous mechanism of 


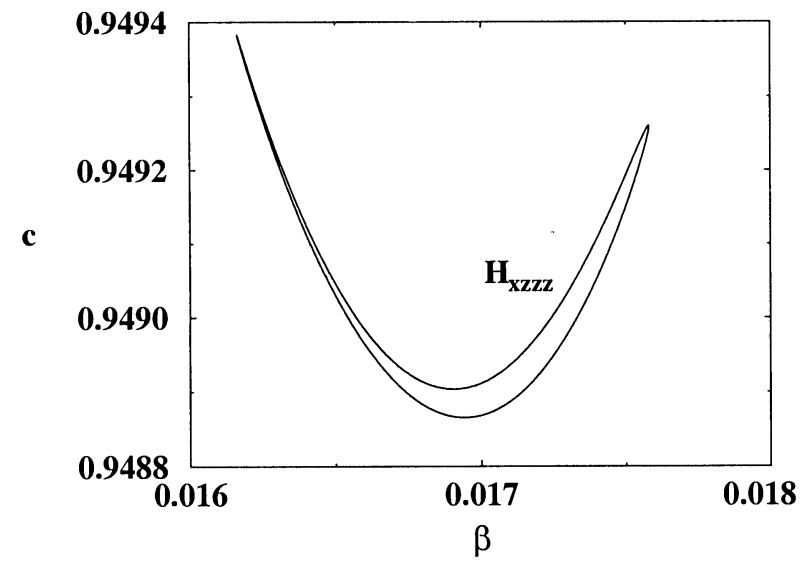

(a)

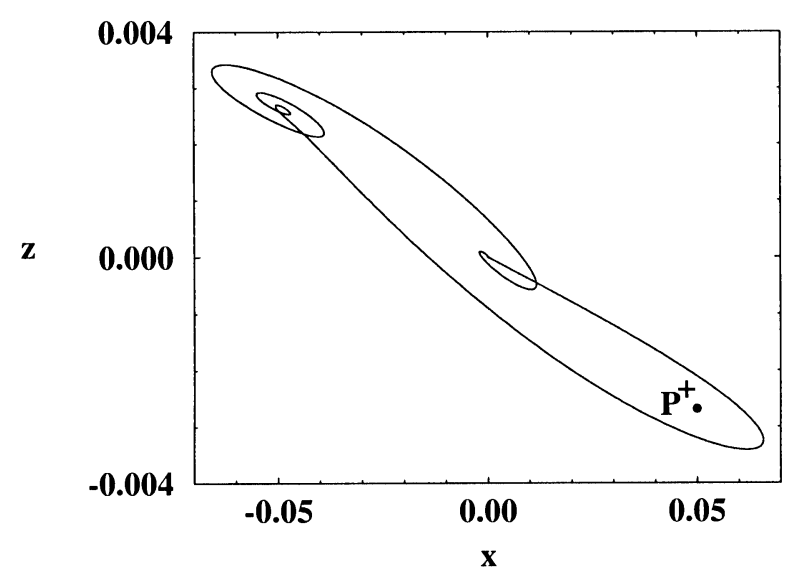

(c)

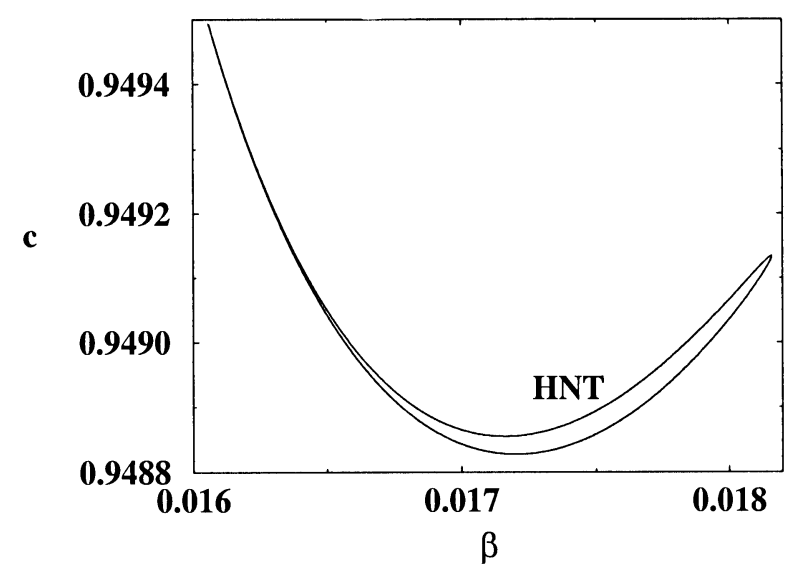

(b)

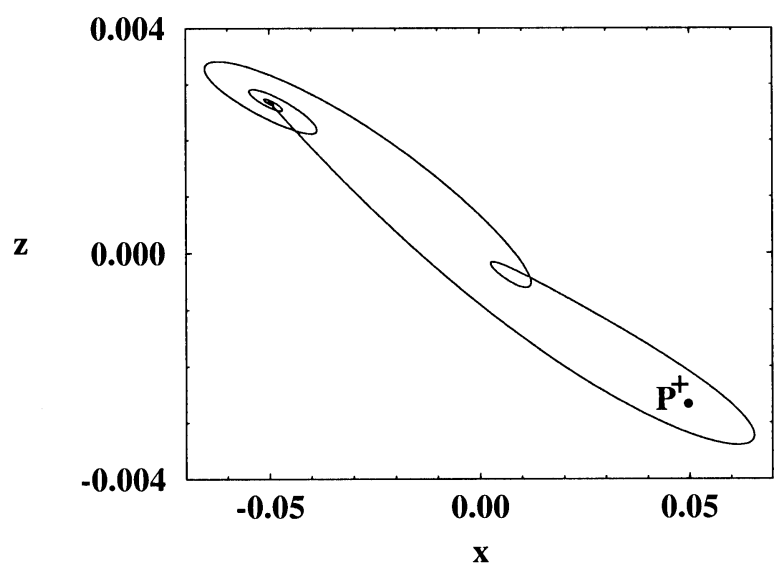

(d)

Fig. 8. For $\alpha=-1.342, \gamma=0.3, a=-1$ : (a) Closed bifurcation curve of homoclinic connections of the origin Hxzzz. (b) Closed bifurcation curve of homoclinic connections of the nontrivial equilibria HNT. (c) Projection onto the $x-z$ plane of the homoclinic connection Hxzzz that occurs for $\beta=0.017, c \approx 0.94887$. (d) Projection onto the $x-z$ plane of the homoclinic connection HNT that occurs for $\beta=0.017, c \approx 0.94884$.

formation, closed curves of both homoclinic connections also occur in the vicinity of a nontransversal T-point. For $\alpha=-1.342$, we show in Figs. 8(a) and 8(b) a closed curve of homoclinic connections of the origin Hxzzz (the projection onto the $x-z$ plane of the one-dimensional manifold of the equilibrium surrounds first $P^{+}$and then three times $P^{-}$) and another one corresponding to homoclinic orbits to the nontrivial equilibria HNT, respectively. Note that both coexist with the closed curve He shown in Fig. 3 (in particular, the closed curve HNT remains inside the closed curve $\mathbf{H e}$, because they cannot intersect). The projection onto the $x-z$ plane of one of the homoclinic connections Hxzzz and HNT appears in Figs. 8(c) and 8(d), respectively.

\section{Conclusions}

Closed curves in bifurcation diagrams (isolas) or in bifurcation sets usually indicate the presence of a higher codimension bifurcation. Several examples have been found in the literature corresponding to bifurcations of periodic orbits.

In this work the presence of closed bifurcation curves of global connections (homoclinic and heteroclinic orbits to equilibria) has been detected in Chua's equation. We have found and described the mechanism of the formation/destruction of such closed curves (sketched in Fig. 7) and have related them to a failure of transversality in a curve of $\mathrm{T}$-points in a three-dimensional parameter space. 
Two open questions are addressed in other work [Algaba et al., 2002]. First, the confirmation of the existence of this phenomenon in other three-dimensional systems. Secondly, the construction of a theoretical model that explains this behavior and confirms our conjecture about the presence of infinitely many closed curves of global bifurcations at the fold point in the curve of T-points in a three-dimensional system.

\section{Acknowledgments}

This work has been partially supported by the Comisión Interministerial de Ciencia y Tecnología in the frame of the project PB98-1152, by the Ministerio de Ciencia y Tecnología, Plan Nacional $I+D+I$, in the frame of the project BFM2001-2608 and by the Consejería de Educación y Ciencia de la Junta de Andalucía (TIC-0130, FQM-276).

\section{References}

Algaba, A., Merino, M., Freire, E., Gamero, E. \& Rodríguez-Luis, A. J. [2000] "On the Hopf-pitchfork bifurcation in the Chua's equation," Int. J. Bifurcation and Chaos 10, 291-305.

Algaba, A., Merino, M. \& Rodríguez-Luis, A. J. [2001] "Takens-Bogdanov bifurcations of periodic orbits and Arnold's tongues in a three-dimensional electronic model," Int. J. Bifurcation and Chaos 11, 513-531.

Algaba, A., Fernández-Sánchez, F., Freire, E. \& Merino, M. \& Rodríguez-Luis, A. J. [2002] "Nontransversal curves of T-points: A source of closed curves of global bifurcations," Phys. Lett. A303, 204-211.

Algaba, A., Merino, M., Freire, E., Gamero, E. \& Rodríguez-Luis, A. J. [2003] "Some results on Chua's equation near a triple-zero linear degeneracy," Int. J. Bifurcation and Chaos 13(3), 583-608.

Bykov, V. V. [1993] "The bifurcations of separatrix contours and chaos," Physica D62, 290-299.
Champneys, A. R. \& Kuznetsov, Y. A. [1994] "Numerical detection and continuation of codimension-two homoclinic bifurcations," Int. J. Bifurcation and Chaos 4, $785-822$.

Champneys, A. R. \& Rodríguez-Luis, A. J. [1999] "The non-transverse Shil'nikov-Hopf bifurcation; uncoupling of homoclinic orbits and homoclinic tangencies," Physica D128, 130-158.

Doedel, E. J., Keller, H. B. \& Kernévez, J. P. [1991] "Numerical analysis and control of bifurcation problems. I. Bifurcation in finite dimensions," Int. J. Bifurcation and Chaos 1, 493-520.

Doedel, E. J., Champneys, A. R., Fairgrieve, T. F., Kuznetsov, Y. A., Sandstede, B. \& Wang, X. [1998] "AUTO97: Continuation and bifurcation software for ordinary differential equations (with HomCont)".

Fernández-Sánchez, F., Freire, E. \& Rodríguez-Luis, A. J. [1997] "Isolas, cusps and global bifurcations in an electronic oscillator," Dyn. Stab. Syst. 12, 319-336.

Fernández-Sánchez, F., Freire, E. \& Rodríguez-Luis, A. J. [2002] "T-points in a $\mathbb{Z}_{2}$-symmetric electronic oscillator. (I) Analysis," Nonlin. Dyn. 28, 53-69.

Glendinning, P. [1984] "Bifurcations near homoclinic orbits with symmetry," Phys. Lett. A103, 163-166.

Glendinning, P. \& Sparrow, C. [1986] "T-points: A codimension-two heteroclinic bifurcation," J. Statist. Phys. 43, 479-488.

Guckenheimer, J. \& Holmes, P. J. [1983] Nonlinear Oscillations, Dynamical Systems, and Bifurcations of Vector Fields (Springer-Verlag, NY).

Hirschberg, P. \& Laing, C. [1995] "Successive homoclinic tangencies to a limit cycle," Physica D89, 1-14.

Kuznetsov, Yu. [1995] Elements of Applied Bifurcation Theory (Springer-Verlag, NY).

Nayfeh, A. H. \& Balachandran, B. [1995] Applied Nonlinear Dynamics (John Wiley, NY).

Pivka, L., Wu, C. W. \& Huang, A. [1996] "Lorenz equation and Chua's equation," Int. J. Bifurcation and Chaos 6, 2443-2489.

Wiggins, S. [1996] Introduction to Applied Dynamical Systems and Chaos (Springer-Verlag, NY). 